\title{
Liver Transplantation in Primary Biliary Cirrhosis: Risk Assessment and 11-Year Follow-Up
}

\author{
Christian Rust $^{a}$ Horst Rau ${ }^{b}$ Alexander L. Gerbes ${ }^{a}$ Gerd R. Pape ${ }^{a}$ \\ Mathias Haller $^{c}$ Hans-Jörg Krämling ${ }^{b}$ Friedrich W. Schildberg ${ }^{b}$ \\ Gustav Paumgartnera Ulrich Beuers ${ }^{a}$
}

Departments of alnternal Medicine II, bSurgery, and 'Anesthesiology, Klinikum Grosshadern, University of Munich, Germany

\section{Key Words}

Liver transplantation - Primary biliary cirrhosis •

Prognostic models · Survival

\begin{abstract}
Background/Aims: Liver transplantation (LTX) is the only established treatment in patients with end-stage primary biliary cirrhosis (PBC). Although short-term survival after LTx in this group of patients is usually good, few data exist on the long-term survival. The optimal timing of transplantation is difficult. Thus, the aims of this study were to assess the long-term survival of patients with PBC after LTx and to identify potential predictive factors for a positive outcome. Methods: Survival of 28 patients with PBC who underwent LTx between 1985 and July 1999 in a single center was studied by Kaplan-Meier analysis and was compared to predicted survival without LTx using established prognostic models for PBC, the Mayo and European risk scores. Potential prognostic parameters obtained before LTx were tested for correlation to survival. Rates of bone fractures as markers of hepatic osteodystrophy were compared before and after LTx. Results: Median follow-up after LTx was 90 months with a maximum of 140 months. Actuarial survival of
\end{abstract}

\begin{tabular}{ll}
\hline KARGER & ( 2000 S. Karger AG, Basel \\
Fax +4161306 1234 & Ac12-2823/00/0621-0038\$17.50/0 \\
$\begin{array}{l}\text { E-Mail karger@karger.ch } \\
\text { www.karger.com }\end{array}$ & $\begin{array}{l}\text { Accessible online at: } \\
\text { www.karger.com/journals/dig }\end{array}$
\end{tabular}

patients with PBC was $89 \%$ after 1,5 , and 10 years and was significantly better than estimated survival without LTx after 1-7 years as calculated by the Mayo and European risk scores. Of several parameters tested, only serum bilirubin and the prognostic scores, but no other liver function tests obtained immediately prior to transplantation were significantly correlated with survival after LTx. The duration of intensive care after LTx was not associated with any parameters obtained before LTx. Bone fractures were diagnosed in $43 \%$ of patients of whom the vast majority were osteopenic before LTx as determined by osteodensitometry. Conclusion: Longterm survival of a well-defined group of patients with PBC was excellent after LTx and was inversely correlated with preoperative serum bilirubin levels as well as Mayo and European risk scores.

Copyright $@ 2000$ S. Karger AG, Basel

\section{Introduction}

Primary biliary cirrhosis (PBC) is the most frequent chronic cholestatic liver disease in adults with a prevalence between 2 and 24 per 100,000 [1]. Though immune mechanisms have been associated with the characteristic

\footnotetext{
Ulrich Beuers, MD

Klinikum Grosshadern, Department of Medicine II

Marchioninistrasse 15, D-81377 Munich (Germany)

Tel. +49 8970953131 , Fax +49 8970958887

E-Mail beuers@med2.med.uni-muenchen.de
} 
histological finding of nonsuppurative destructive cholangitis [2], the pathogenesis of PBC still remains unclear and treatment is difficult. Numerous drugs have been evaluated for the treatment of PBC, but to date only ursodeoxycholic acid has been proven to be a safe and effective drug that may slow the progression of this disease [3]. In the end stage of PBC, liver transplantation (LTx) has been proven to be highly effective [4]. Thus, the identification of risk factors and an estimation of survival with or without LTx for these patients is needed to achieve an adequate timing of LTx [4-7]. Several prognostic indices have been established to predict the probability of survival in patients with $\mathrm{PBC}$, of which the Mayo risk score [6] and the updated European risk score [7] are most widely used. Additional prognostic scores have been introduced to predict the amount of intraoperative blood loss, number of days in the intensive care unit (ICU), and severe complications after surgery [8]. However, optimal timing for LTx in the individual patient with PBC still remains difficult. In addition, the waiting time for LTx is increasing due to the lack of donor organs, and this leads to an increased mortality on the waiting list [9].

The aim of this study was to compare the long-term survival of patients with PBC after LTx to estimated survival without LTx using the Mayo as well as the European prognostic model. In addition, baseline characteristics of the patients were correlated with survival following LTx and with the duration of intensive care. Since osteodystrophy is a major complication of advanced PBC and may aggravate after LTx due to corticosteroid treatment and transient immobilization, rates of bone fractures and bone density were compared before and after LTx.

\section{Patients and Methods}

Between 1985, when the LTx program started at the University of Munich, and the final follow-up on July 1, 1999, 28 patients (27 women, 1 man) with PBC underwent LTx, representing 9\% of all indications for LTx in the program. All patients received AB0 compatible grafts. Cardiovascular diseases, malignancies, infections and concomitant liver diseases were excluded before patients were listed for LTx. Long-term immunosuppression was maintained with cyclosporin or tacrolimus according to blood levels and $5 \mathrm{mg}$ prednisolone equivalent daily. Patient data from the last follow-up visit before LTx (range 2 days to 2 months before LTx) were used for the calculation of risk scores. After LTx, patients were followed on a yearly basis as outpatients by physicians of the transplant program and were readmitted to the inpatient service for all LTx-related complications during follow-up. No patient was lost to follow-up.

The median age of the 27 women and 1 man with PBC at transplantation was 54 (36-65). Median follow-up after LTx was 90 months with a maximum of 140 months. At transplantation, $45 \%$ of
Table 1. Baseline characteristics of patients with PBC before LTx $(n=28)$

\begin{tabular}{lcc}
\hline Characteristic & Median & Range \\
\hline Age, years & 54 & $36-65$ \\
Bilirubin, mg/dl & 14.9 & $2.5-49.2$ \\
Albumin, g/dl & 3.4 & $2.5-4.2$ \\
Creatinine, mg/dl & 0.9 & $0.6-1.7$ \\
Prothrombin time, $\%$ & 65 & $42-100$ \\
ABT, $\%$ & 0.134 & $0.018-0.608$ \\
Time in the ICU, days & 15 & $1-75$ \\
Mayo model & 8.224 & $6.367-11.016$ \\
European model & 2.581 & $0.321-5.375$ \\
\hline Child-Pugh score ${ }^{1}$ & A (5-6 points) & $0 \%$ \\
& B (7-9 points) & $68 \%$ \\
UNOS status & C ( $\geq 10$ points) & $32 \%$ \\
& 1 (ICU/liver failure) & $0 \%$ \\
& 2 (hospitalized) & $45 \%$ \\
& 3 (continuous care) & $55 \%$
\end{tabular}

Biochemical results were obtained within 2 months prior to LTx.

UNOS = United Network for Organ Sharing.

1 Child-Pugh score was modified for PBC as proposed by Pugh et al. [10].

patients were hospitalized and 55\% received continuous medical care, but were at home. The median serum bilirubin level was 14.9 $(2.5-49.2) \mathrm{mg} / \mathrm{dl}$. Renal function as assessed by serum creatinine levels was normal in 26 and only mildly impaired in 2 of 28 patients. The median creatinine level was $0.9(0.63-1.7) \mathrm{mg} / \mathrm{dl}$. All characteristics of the patient population are summarized in table 1 .

Osteodensitometry was performed in the lumbar spine using CT until July 1991 when QDR was introduced. After July 1991, QDR of the lumbar spine became the method of choice for osteodensitometry due to low radiation exposure and high diagnostic accuracy.

\section{Prognostic Models for Survival}

The actual survival time after LTx was compared to the estimated survival time without LTx as calculated by two prognostic scores for patients with PBC, the Mayo risk score and the European risk score $[6,7]$. These models were chosen, because they are crossvalidated and do not require inclusion of histological parameters for estimation of prognosis. Both models are based on Cox's proportional hazards model and can be used to calculate a prognostic index for any patient at a given time.

The Mayo model described by Dickson et al. [6] includes five variables which independently predict survival in PBC. The prognostic index $R$ is calculated from these variables using the following equation: $\mathrm{R}=0.871 \times \log _{\mathrm{e}}($ bilirubin $[\mathrm{mg} / \mathrm{dl}])-2.53 \times \log _{\mathrm{e}}($ albumin $[\mathrm{g} / \mathrm{dl}])+0.039 \times$ age $($ years $)+2.38 \times \log _{\mathrm{e}}($ prothrombin time $[\mathrm{s}])+$ $0.859 \times$ edema $(0=$ no edema, no diuretic therapy; $0.5=$ edema, no diuretic therapy or no edema, diuretic therapy; 1 = edema and diuretic therapy). 
Fig. 1. Actuarial and estimated survival of patients with PBC. Actuarial survival of patients with PBC after LTx (Kaplan-Meier) was significantly better $(\mathrm{p}<0.01-0.001)$ than the estimated survival without LTx as calculated by the Mayo and European prognostic models for time points of 1-7 years after LTx. The number of patients followed is given in parentheses for all time points.

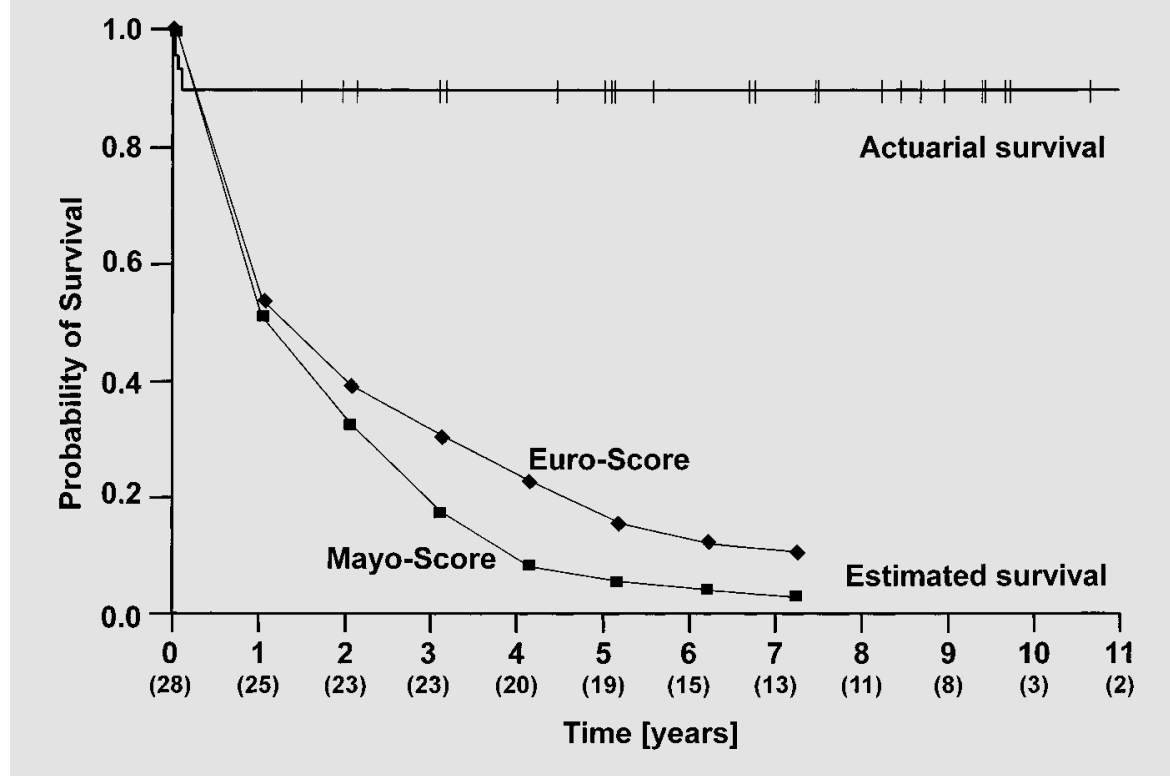

The European model described by Christensen et al. [7] includes six independent prognostic variables. These variables are used to calculate the prognostic index $\mathrm{PI}_{\mathrm{AI}}$ as follows: $\mathrm{PI}_{\mathrm{AI}}=2.53 \times(\log$ bilirubin $[\mu \mathrm{mol} / 1]-1.53)+1.39 \times$ ascites $(0=$ no; $1=$ yes $)-0.089 \times$ (albumin $[\mathrm{g} / \mathrm{l}]-34.3)+0.04 \times($ age [years] -55$)+0.65 \times$ GI bleeding $(0=$ no; $1=$ yes $)$.

\section{Prognostic Model for Duration of ICU Stay}

Recently, a prognostic score has been proposed to predict the number of days in the ICU after LTx for patients with cholestatic liver disease [8]. ICU score $=-48+(1.1 \times$ age [years] $)+(63 \times$ renal failure $[0=$ no; $1=$ yes $])+(25 \times$ Child's class $[\mathrm{A}=1 ; \mathrm{B}=2 ; \mathrm{C}=3])-$ $(19 \times$ UNOS status $[1=$ ICU/liver failure; $2=$ hospitalized; $3=$ continuous care; $4=$ at home]). In this model, renal failure is defined as serum creatinine $>2.0 \mathrm{mg} / \mathrm{dl}$ and/or urine output $<10 \mathrm{ml} / \mathrm{kg} / 24 \mathrm{~h}$. A modified Child's classification for patients with cholestatic liver disease was used to calculate the Child-Pugh score [8].

\section{Statistical Methods}

Survival time was defined as the interval between the first LTx and death or final follow-up (July 1, 1999). Retransplantation performed in 3 patients due to graft failure was disregarded because patient survival, not graft survival was analyzed. Thus, each of these patients was included in the study only once and the time point of the initial transplantation was used for the follow-up. Survival was calculated using the Kaplan-Meier analysis. Estimated survival time for patients without LTx was calculated as described above [6, 7]. The difference between estimated and actuarial survival was analyzed by using the $\chi^{2}$ test. Correlation of baseline characteristics to survival and duration of time on an ICU was tested by using Wilcoxon's test for unpaired samples. A $p<0.05$ was considered statistically significant.

\section{Results}

\section{Survival}

Of 28 patients who underwent LTx for PBC, 25 (89\%) were alive at the endpoint of follow-up; median follow-up was 90 months with a maximum of 140 months. All lethal outcomes were related to early mortality: 3 patients died within 1 week of transplantation, 1 due to primary graft dysfunction, and two due to bleeding complications. These 3 patients also had advanced disease as indicated by a Child-Pugh score of 11,11 , and 10 points, respectively. The actuarial survival rate after LTx was $89 \%$ from year 1 to 7 using the Kaplan-Meier estimate, and higher than the estimated survival rate using the Mayo as well as the European prognostic model (fig. 1). These differences between the actuarial survival and the estimated survival without LTx were highly significant for all time points (year 1-7) and both models used.

Several parameters of potential prognostic value were tested for a possible association with the outcome of LTx in our patient population. Of these parameters, preoperative serum bilirubin levels $(\mathrm{p}<0.001)$ as well as the European and Mayo prognostic scores $(\mathrm{p}<0.05$, each) were significantly correlated with survival after LTx (fig. 2). In contrast, age, albumin, prothrombin time and hepatic microsomal demethylation capacity as determined by the aminopyrine breath test (ABT; fig. 2) were not significantly associated with survival. 
Fig. 2. Prognostic factors for survival after LTx. Serum bilirubin, Mayo and European risk scores, and aminopyrine breath test (ABT) of patients with PBC obtained within 2 months prior to LTx. $O=$ Values of patients surviving LTx; $>$ patients who died after LTx. Differences between values of surviving and nonsurviving patients were statistically significant for serum bilirubin $(\mathrm{p}<$ $0.001)$, Mayo $(\mathrm{p}<0.01)$ and European risk score $(\mathrm{p}<0.01)$, but not for the ABT.

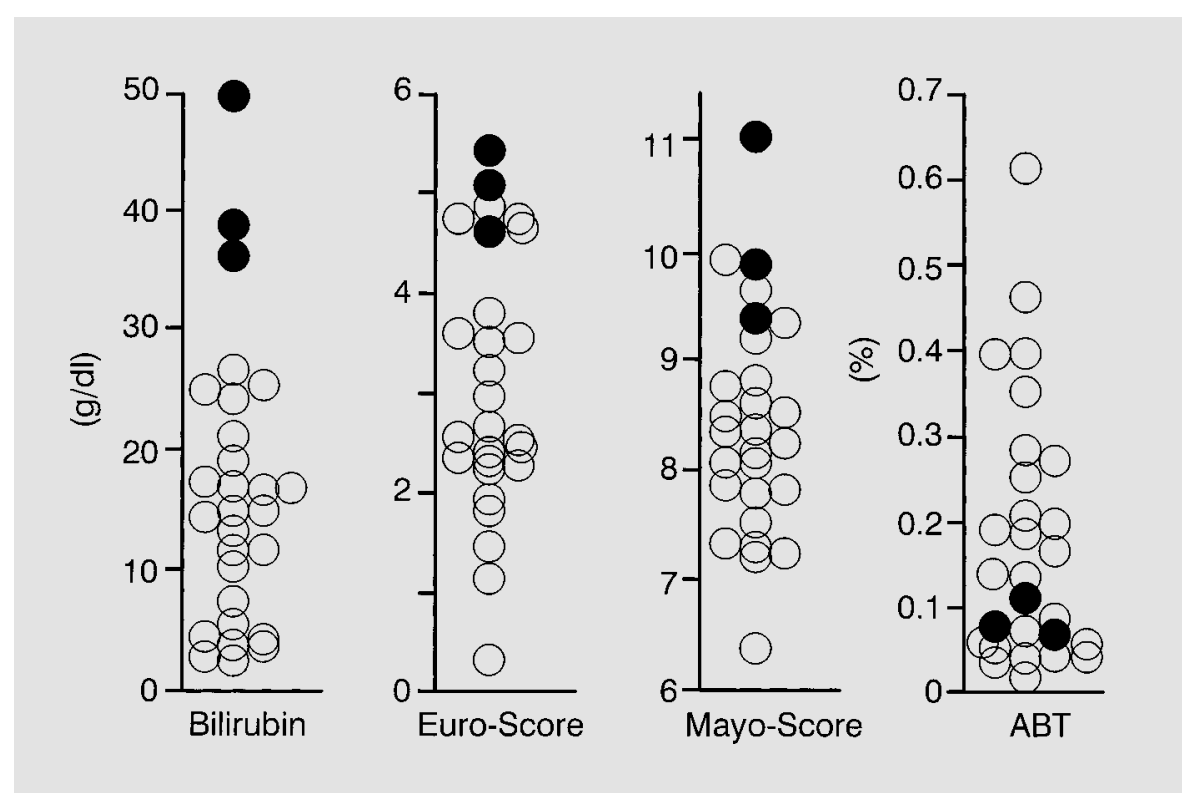

\section{Duration of Intensive Care}

After LTx, patients were treated for a median of 15 (1-75) days in an ICU. The 3 patients who died after transplantation were disregarded for these calculations. No correlation was found between potential prognostic variables determined before LTx (bilirubin, prothrombin time, age, albumin, ABT, Mayo and European score) and the duration of intensive care. In addition, a prognostic score proposed by Ricci et al. [8] to estimate the number of days on an ICU after LTx did not predict the period of intensive care in our patient population when the actual days on the ICU were compared to the putative number of days as calculated by this model. However, the median stay in an ICU was only 3 days in the study population of Ricci et al. [8], whereas we observed a median stay of 15 days.

\section{Osteodystrophy}

Bone fractures are the major manifestation of hepatic osteodystrophy in patients with PBC and may lead to disability before as well as after LTx. In our unit, patients were receiving calcium supplementation (1,000 mg daily) before and after LTx. In addition, patients were also supplemented with vitamin $\mathrm{D}_{3}$ (cholecalciferol 0.025-0.050 mg equivalent to $1,000-2,000$ IU vitamin $\mathrm{D}_{3}$ daily), when their serum level of 25-hydroxycholecalciferol was in the low normal range or below the normal range. Bone fractures mainly of lumbar and thoracic vertebrae were diagnosed in $32 \%$ of patients before LTx. All these patients had a bone density (as determined in the lumbar spine) of more than 1 SD below that of healthy women of the same age. Another $25 \%$ of patients who presented with bone densities lower than 1 SD below that of healthy controls did not develop fractures before LTx. However, one or more new fractures were observed in $28 \%$ of patients surviving LTx (first fracture in 11\%). Again, fractures mainly affected the vertebrae and all but 1 patient had a bone density of more than 1 SD below the bone density of healthy controls. Taken together, osteodystrophy was a common complication and led to bone fractures in $43 \%$ of patients under study before and/or after transplantation.

\section{Discussion}

This study shows that the beneficial effect of LTx on survival of patients with PBC is maintained for a median follow-up of 90 months and a maximum survival of more than 11 years, the longest follow-up reported so far. LTx significantly improved the long-term survival of patients with PBC when compared to predicted survival without transplantation. The cumulative survival of $89 \%$ observed up to 11 years after LTx appears remarkable and is comparable or superior to the 5-year patient survival reported previously [11-15].

Survival of patients with PBC has been less favorable in previous decades although patient characteristics were comparable. In addition, follow-up time is shorter in most 
papers. Markus et al. [12] followed 161 patientswith PBC after LTx between 1980 and 1987. Baseline characteristics were similar to our patients with regard to serum bilirubin, prothrombin time and Mayo risk score, but with a median of 47.8 years these patients were on average 7 years younger at transplantation. The median follow-up was 25 months and the actuarial survival 1 year after LTx was $76 \%$. Keiding et al. [11] followed 137 patients, who received LTx for PBC in Nordic countries between 1982 and 1988 for a median of 13 months. Actuarial survival after 1 year was $75 \%$ in their study population. Bonsel et al. [13] followed 30 patients with PBC after LTx between 1978 and 1989. The patients of their study were younger (median 49.6 years), serum bilirubin was lower (median $11.7 \mathrm{~g} / \mathrm{dl}$ ) and median follow-up was 30 months with a maximum of 9.9 years. Despite the favorable baseline characteristics, cumulative survival was only $65 \%$ after 1 year and about $60 \%$ after 5 years. These differences in survival time when compared to our study might be explained by advances in perioperative and posttransplantation patient management during the last 10-20 years.

More recently, Knoop et al. [14] reported a 5-year survival rate of $95 \%$ in 49 patients with PBC. The median preoperative serum bilirubin was $8.9 \mathrm{~g} / \mathrm{dl}$ as compared to $14.9 \mathrm{~g} / \mathrm{dl}$ in our study, suggesting less advanced disease stages in their group of patients.

A Mayo risk score of 7.8 and higher has been associated with a progressively increased mortality after LTx [15]. However, $71 \%$ of our patients had a Mayo risk score greater than 7.8. Thus, LTx may be successful with Mayo risk scores of considerably more than 7.8, and additional parameters and risk scores should be included and evaluated for the optimal timing of LTx in patients with PBC. Serum bilirubin has been shown to be a potent predictor of survival in untreated patients with PBC [16-18]. In our study, pretransplant serum bilirubin levels were significantly associated with survival after LTx confirming a previous report [15]. Indeed, patients with pretransplant serum bilirubin levels above $27 \mathrm{~g} / \mathrm{dl}$ did not survive the first week after LTx. Prospective evaluation of the prognostic value of serum bilirubin for the outcome of LTx in $\mathrm{PBC}$ is clearly of interest. The European and Mayo prognostic models, each including serum bilirubin, were also correlated to survival. Age has been inversely related to survival in untreated patients with PBC [5-7], but was not correlated to survival after LTx in our study. The hepatic demethylation capacity as determined by the ABT was not associated with survival after LTx in the patients studied. However, the ABT is of limited value in the assessment of disease severity in patients with cholestasis [19]. The lim- ited number of patients in our study may have precluded the identification of additional factors potentially predictive for the outcome of LTx in PBC.

The duration of intensive care was not correlated to any of the potential prognostic factors studied in our patients. In addition, a prognostic model proposed to predict the duration of intensive care after LTx in patients with cholestatic liver disease could not be applied to our patients. However, Ricci et al. [8] reported a median period on ICU of 3 days compared to a median of 15 days in our study. Thus, the model of Ricci et al. [8] might not be applicable to the conditions met in most transplantation centers in Germany where patients are generally observed for a longer time period on an ICU after LTx.

Osteoporosis and atraumatic fractures are serious complications of PBC and may considerably worsen a patient's general condition after LTx [20]. Female patients with PBC have a reduced mean bone density when compared to healthy women [21], resulting in atraumatic fractures before and shortly after transplantation. In our study, $57 \%$ of all patients had a decreased bone density and more than half of these patients, equaling $32 \%$ of the whole study population, developed one or more fractures prior to transplantation. These numbers highlight the fact that osteodystrophy is common in PBC patients and fractures are a major complication before LTx. Bone density should, therefore, be determined in all patients and preventive treatment started early in the course of disease. After LTx, 28\% of the surviving patients were diagnosed with new bone fractures. All but one of these patients again had decreased bone density, even though it has been shown that bone mass may be restored towards normal within 2-3 years after LTx [21]. However, all but 1 of our patients were treated with low-dose corticosteroids after LTx. This patient had severe osteodystrophy before transplantation with fractures of several lumbar and thoracic vertebrae. A long-term immunosuppression without corticosteroids was chosen for this patient to prevent prednisolone-induced aggravation of bone loss after LTx. Thus, treatment of osteodystrophy appears necessary before and after LTx in the majority of patients with PBC. Recently, cyclical etidronate has been used successfully to prevent bone loss in corticosteroid-treated PBC patients. Bisphosphonates might provide a new treatment strategy for this group of patients [22].

In summary, LTx proved to be beneficial in a group of patients with PBC followed for a period of more than 10 years. Serum levels of bilirubin and multiparametric prognostic indices were correlated to the outcome of LTx in our patient group. $\overline{42} \quad \overline{\text { Digestion 2000;62:38-43 }}$
Rust/Rau/Gerbes/Pape/Haller/Krämling/ Schildberg/Paumgartner/Beuers 


\section{References}

1 Neuberger J: Primary biliary cirrhosis. Lancet 1997;350:875-879.

2 Kaplan MM: Primary biliary cirrhosis. New Engl J Med 1996;335:1570-1580.

3 Poupon RE, Lindor KD, Cauch-Dudek K, Dickson ER, Poupon R, Heathcote EJ: Combined analysis of randomized controlled trials of ursodeoxycholic acid in primary biliary cirrhosis. Gastroenterology 1997;113:884-890.

4 Benhamou JP: Indications for liver transplantation in primary biliary cirrhosis. Hepatology 1994;20(suppl):11-13.

5 Beuers U, Paumgartner G: Assessment of treatment in cholestasis; in Poupon $\mathrm{R}$, Reichen $\mathrm{J}$ (eds): Surrogate Markers to Assess Efficacy of Treatment in Chronic Liver Diseases. Lancaster, Kluwer Academic, 1996, pp 153-163.

6 Dickson ER, Grambsch PM, Fleming TR, Fisher LD, Langworthy A: Prognosis in primary biliary cirrhosis: Model for decision making. Hepatology 1989;10:1-7.

7 Christensen E, Altman DG, Neuberger J, De Stavola BL, Tygstrup N, Williams R: Updating prognosis in primary biliary cirrhosis using a time-dependent Cox regression model. PBC1 and PBC2 trial groups. Gastroenterology 1993; 105:1865-1876.

8 Ricci P, Therneau TM, Malinchoc M, Benson JT, Petz JL, Klintmalm GB, Crippin JS, Wiesner RH, Steers JL, Rakela J, Starzl TE, Dickson ER: A prognostic model for the outcome of liver transplantation in patients with cholestatic liver disease. Hepatology 1997;25: 672-677.
9 Everhart JE, Lombardero M, Detre KM, Zetterman RK, Wiesner RH, Lake JR, Hoofnagle $\mathrm{JH}$ : Increased waiting time for liver transplantation results in higher mortality. Transplantation 1997;64:1300-1306.

10 Pugh RN, Murray-Lyon IM, Damson IL, Pietroni MC, Williams R: Transection of the oesophagus for bleeding oesophageal varices. $\mathrm{Br} \mathrm{J}$ Surg 1973;60:646-649.

11 Keiding S, Ericzon BG, Eriksson S, Flatmark A, Hockerstedt $\mathrm{K}$, Isoniemi $\mathrm{H}$, Karlberg I, Keiding N, Olsson R, Samela K, Schrumpf E, Söderman C: Survival after liver transplantation of patients with primary biliary cirrhosis in the Nordic countries. Comparison with expected survival in another series of transplantations and in an international trial of medical treatment. Scand J Gastroenterol 1990;25:1118.

12 Markus BH, Dickson ER, Grambsch PM, Fleming TR, Mazzaferro V, Klintmalm GB, Wiesner RH, Van Thiel DH, Starzl TE: Efficiency of liver transplantation in patients with primary biliary cirrhosis. N Engl J Med 1989; 320:1709-1713.

13 Bonsel GJ, Klompmaker IJ, van't Veer F, Habbema JD, Slooff MJ: Use of prognostic models for assessment of value of liver transplantation in primary biliary cirrhosis. Lancet 1990;335: 493-497.

14 Knoop M, Bechstein WO, Schrem H, Lobeck $\mathrm{H}$, Hopf U, Neuhaus P: Clinical significance of recurrent primary biliary cirrhosis after liver transplantation. Transplant Int 1996;9(suppl 1):115-119.

15 Kim WR, Wiesner RH, Therneau TM, Poterucha JJ, Porayko MK, Ebans RW, Klintmalm GB, Crippin JS, Krom RA, Dickson ER: Optimal timing of liver transplantation for primary biliary cirrhosis. Hepatology 1998;28:33-38.
16 Roll J, Boyer JL, Barry D, Klatskin G: The prognostic importance of clinical and histologic features in asymptomatic and symptomatic primary biliary cirrhosis. New England Journal of Medicine 1983; 308:1-7.

17 Shapiro JM, Smith H, Schaffner F: Serum bilirubin: A prognostic factor in primary biliary cirrhosis. Gut 1979;20:137-140.

18 Neuberger J, Altman DG, Polson R, Buckels J, Rolles K, Elias E, Calne R, McMaster P, Williams R: Prognosis after liver transplantation for primary biliary cirrhosis. Transplantation 1989;48:444-447.

19 Baker AL, Krager PS, Kotake AN, Schoeller DA: The aminopyrine breath test does not correlate with histologic disease severity in patients with cholestasis. Hepatology 1987;7: 464-467.

20 Hodgson SF, Dickson ER, Wahner HW, Johnson KA, Mann KG, Riggs BL: Bone loss and reduced osteoblast function in primary biliary cirrhosis. Ann Intern Med 1985; 103:855-860.

21 Eastell R, Dickson ER, Hodgson SF, Wiesner RH, Porayko MK, Wahner HW, Cedel SL, Riggs BL, Krom RA: Rates of vertebral bone loss before and after liver transplantation in women with primary biliary cirrhosis. Hepatology 1991;14:296-300.

22 Wolfhagen FH, van Buuren HR, den Ouden JW, Hop WC, van Leeuwen JP, Schalm SW, Pols HA: Cyclical etidronate in the prevention of bone loss in corticosteroid-treated primary biliary cirrhosis. A prospective, controlled pilot study. J Hepatol 1997;26:325-330. 